\title{
Gradient, counter-gradient transport and their transition in turbulent premixed flames
}

\author{
Vladimir Zimont† and Fernando Biagiolił $\$$ \\ $\dagger$ CRS4, Sesta Strada Ovest, Z I Macchiareddu, I-09010 Uta (Ca), Italy \\ $\div$ ALSTOM Power UK Ltd, PO Box 1, Waterside South, Lincoln LN5 7FD, UK \\ E-mail: zimont@crs4.it, fernando.biagioli@power.alstom.com
}

\begin{abstract}
We theoretically and numerically analyze the phenomenon of countergradient transport in open premixed turbulent flames. The focus is on the transition from counter-gradient to gradient transport obtained when reducing the turbulence intensity/laminar flame speed ratio, a phenomenon recently found in open laboratory flames experiments by Frank et al. The analysis is based on the TFC combustion model for the simulation of turbulent premixed flames at strong turbulence $\left(u^{\prime}>>s_{L}\right)$. In this case earlier work suggests that turbulent premixed flames have increasing flame brush width controlled in the model only by turbulence and independent from the counter-gradient transport phenomenon which has gasdynamics nature, and a turbulent flame speed which quickly adapts to a local equilibrium value, i.e. Intermediate Steady Propagation (ISP) flames. According to the present analysis transport in turbulent premixed flames is in fact composed by two contributions: real physical gradient turbulent diffusion, which is responsible for the growth of flame brush thickness, and counter-gradient pressure-driven convective transport related to the differential acceleration of burnt and unburnt gases subject to the average pressure variation across the turbulent flame. The novel gas dynamics model for the pressuredriven transport which is developed here, shows that in open turbulent premixed flames the overall transport may be of gradient or counter-gradient nature according to which of these two contributions is dominant and that along the flame a transformation from gradient to counter-gradient transport takes place. Reasonable agreement with the mentioned laboratory experimental data, strongly support the validity of the present modelling ideas. Finally, the model predicts existence of this phenomenon also in large-scale industrial burners at much higher Reynolds numbers.
\end{abstract}

Submitted to: Combust. Theory Modelling

$\S$ To whom correspondence should be addressed (fernando.biagioli@power.alstom.com) 


\section{Introduction}

Counter-gradient transport is a phenomenon which commonly occurs in turbulent premixed combustion. It is connected with the differential acceleration of hot and cold fluid volumes under the flame pressure drop generated by heat release. It has been experimentally observed for example in open [1] and impinging [2] flames. This phenomenon is closely connected with the flamelets combustion mechanism in turbulent premixed flames, when combustion takes place in thin and strongly wrinkled layers called flamelet sheets. In laboratory flows with relatively low turbulence Reynolds numbers $R e_{t}=u^{\prime} L / \nu$ these flamelet sheets are laminar flames with speed $s_{L}$ and thickness $\delta_{L}$ highly wrinkled by small scale turbulence.

A new phenomenon consisting in the transition from counter-gradient to gradient transport has been recently experimentally observed [3]. According to these experiments in open premixed flames, counter-gradient transport observed at some fixed distance from the burner inlet transforms into gradient transport when the ratio between the turbulent velocity fluctuation $u^{\prime}$ and the laminar flame speed $s_{L}$ increases as consequence of the variation in the fuel/air mixture equivalence ratio. In the present paper we focus the main attention on the theoretical analysis of this phenomenon, its numerical simulation and comparison with these laboratory experimental data.

Another issue addressed in the paper is the existence of counter-gradient transport and the phenomenon of transition to gradient transport in large-scale high velocity flames in industrial combustors where direct measurements are very difficult. Our point of view in fact is that in industrial burners characterised by much larger Reynolds and Damköhler ( $D a=\tau_{t} / \tau_{c h}$ ratio between the integral turbulence and chemical times scales) numbers, presence of counter-gradient transport is highly probable. At large turbulent Reynolds numbers in fact, turbulence has a very wide continuous spectrum of eddies including very small dissipative vortices. At Damköhler numbers in industrial burners $(D a \simeq 10)$ the size of the smallest Kolmogorov eddies $\eta=L R e_{t}^{-3 / 4}$ (where $L$ is the integral length scale of turbulence) can be comparable or less than the laminar flame thickness, i.e. $\eta \leq \delta_{L}$. In this case we have wrinkled flamelets which can have thickness $\delta_{f}$ larger than the laminar one $\delta_{L}$ because of the intensification in the transport processes by small-scale eddies with size less than $\delta_{f}$. Furthermore, if the turbulent integral length scale $L$ is much larger that $\delta_{f}$, it means that combustion takes place in a strongly wrinkled sheet that is not laminar but appears broadened by small scale turbulence. It must be mentioned also that turbulent stretch can reduce the width of these flamelets incorporating fine scale vortices. Therefore, generally speaking, the actual width of these flamelets thickened by fine scale turbulence can be even less than the width of an unstretched laminar flame (but obviously larger than the correspondent stretched laminar flame width).

As the counter-gradient phenomenon is closely connected with the flamelet combustion mechanism, it is important to stress that the successive falling of always larger eddies in the expanding broadened flamelet has a limit. Equilibrium thickness 
is in fact achieved when convection, diffusion and heat release intensity have the same order of magnitude [4, 5]. Such expanded equilibrium flamelet, in accordance with the theoretical estimations at $D a>>1$, is always characterized by $\delta_{f}>\delta_{L}$. Existing direct experimental data $[6,7]$ demonstrate that flamelets thickness is slightly larger $(3-5$ times) than the stretched laminar flame thickness (tough smaller than the thickness of the normal 1D laminar flame), a typical estimation being of the order of $1 \mathrm{~mm}$.

Unfortunately, there is no direct evidence that combustion takes place in thin and strongly wrinkled sheets also in industrial combustors, where $u^{\prime}$ can be quite large, as there are not direct measurements of the flamelet parameters in these kind of burners. In spite anyway of the increase in the ratio $\delta_{f} / L$ for increasing $u^{\prime}$ (which gives a decrease in $D a)$, estimations presented in [5] show that extinction due to flamelet stretch takes place long before the formation of distributed combustion zones with $\delta_{f} / L \geq 1$. We therefore believe that counter-gradient transport and the phenomenon of transition to gradient transport exist also in flames in industrial burners. We must nevertheless mention that a different point of view, which we do not share, is often found in literature. According to this, industrial combustion occurs with the distributed volume mechanism corresponding to the model of stirred reactor. Clearly, in this case a well-defined counter-gradient phenomenon is not likely to be possible.

The effect of small-scale turbulence with size larger than $\delta_{f}$ is to make the flamelet sheet wrinkled. This increases the flamelet surface-turbulent flame cross sectional areas ratio $\bar{S} / S_{0}$ which, together with the expanded flamelet combustion velocity $U_{f}$, controls the turbulent burning velocity, i.e. $U_{t}=U_{f} \bar{S} / S_{0}$. It is very significant to emphasize here that, for the case of fully developed turbulence, the main contribution to the increase in $\bar{S} / S_{0}$ is given by small-scale wrinkles generated by eddies with sizes $\delta_{f} \leq d<<L$, while large-scale wrinkles caused by eddies with $d \sim L$ mainly control the flame brush width and give only relatively small contribution to $\bar{S} / S_{0}$.

The consequence of this situation is that immediately after combustion has started, we have not only equilibrium broadened flamelets with $U_{f}=$ const but also equilibrium small-scale wrinkles structure of the flamelet sheet. On the other hand, in the case of $u^{\prime}>U_{f}$ and during a relatively long period of time, the large scale wrinkles structure will not be in equilibrium (according to estimations in [8] during a time scale of $\left.t \sim \tau_{t}\left(u^{\prime} / U_{f}\right)^{2}\right)$. The practical result of this is that during this relatively long period of time, an intermediate propagation combustion regime, preceeding the formation of a stationary combustion front structure with constant $U_{t}^{s t}$ and $\delta_{t}^{s t}$, takes place. Premixed combustion within this intermediate regime takes place therefore in flames with increasing flame brush width controlled by turbulent diffusion and with practically constant turbulent combustion velocity $U_{t} \sim$ const (more exactly with slowly increasing $U_{t}$ due to relatively small contribution to the flamelet sheet area of nonequilibrium large wrinkles). Strictly speaking this arguments are valid only in the hypothetical case of combustion at constant density. Leaning upon experimental observations, we have postulated the same physical picture and the possibility to use the associated combustion model also in the real case of variable density. 
Prudnikov was probably the first who established this peculiarity of premixed combustion during the late '50s. He in fact showed experimentally that turbulent premixed flames stabilized in uniform duct flows with strong artificial turbulence had temperature profiles corresponding to the integral probability distribution, i.e. close to the normal flamelet probability density function, and flame brush with increasing width which was controlled mainly by the cold flow turbulent diffusion coefficient. This result was first published in 1959 in a nowadays hard-to-find preprint and successively presented in [9].

This particular regime of turbulent premixed combustion has been named Intermediate Steady Propagation (ISP) regime [5, 8] (intermediate asymptotic between the initial stage of formation of an equilibrium small-scale wrinkled structure and final stage of a turbulent flame completely in equilibrium with $U_{t}=$ const and $\delta_{t}=$ const) in order to emphasize the concept of flames characterized by approximately constant turbulent flame speed (constant in the case of homogeneous turbulence and equilibrium in more complex situations) and a flame brush thickness which grows according to the turbulent dispersion law. The reason of ISP flames prevailing within an industrial combustor is given by the the fact that combustion in this burners is still well far from the final stage of fully steady conditions (constant $U_{t}$ and $\delta_{t}$ ) as the mechanism which is responsible for compensating the turbulent growth of the flame brush thickness becomes effective on a time scale much larger than the average residence time.

In the case of open axisymmetric premixed flame analyzed in this paper, this concept means that a premixed flame is simply a turbulent mixing layer with increasing width (similarly to the mixing layer of a non reactive flow), propagating in the fresh mixture with equilibrium combustion velocity $U_{t}$. This propagating mixing layer crosses at the combustor axis long before the formation of the flame with complete equilibrium structure.

Given that a flame in complete equilibrium with $U_{t}=$ const and $\delta_{t}=$ const is unattainable in practical devices under strong turbulence conditions, it is reasonable to develop a turbulent premixed combustion model which describes only the ISP regime and does not contain the mechanisms responsible for reaching the final full equilibrium flame structure. This model, which was proposed and investigated in the papers [4, 5] and called in [10] the Turbulent Flame Closure (TFC) combustion model, has been further developed and used here for the description of counter-gradient and gradient transport as well as their transition in correspondence of variations in the fuel/air mixture equivalence ratio.

The reasons of experimentally observed turbulent premixed flames with increasing width might not be completely clear at this stage, deserving then further comments. A contradiction in fact apparently arises between the idea of a turbulent flame brush which grows in thickness according to the turbulent dispersion law (i.e. according to a positive turbulent diffusion coefficient) and the experimentally observed counter-gradient nature of the progress variable transport which has been so far the subject of many theoretical and numerical studies. For example Moss [1] has observed this phenomenon in his 
early experiments for an open premixed flame; subsequently counter-gradient transport has been observed in the experiments performed in $[2,11]$ and in direct numerical simulations of turbulent premixed flame [12]. It has been consequently argued that the assumption of gradient transport for the progress variable or the reactive components is in general not appropriate for turbulent premixed flames and sometimes also that, as a consequence of "counter-gradient diffusion", the flame brush thickness decreases instead than increasing [12]. The possibility to model the turbulent transport of reactive components in turbulent premixed combustion using models based on gradient transport and a conveniently estimated turbulent diffusion coefficient has been therefore abandoned a long time ago and the attention has been totally concentrated on second order moment closure methods [13, 14].

The point of view developed in this paper is that the term "counter-gradient diffusion" often given in the literature to describe transport of the progress variable in flows with heat release has its origin in the interpretation of this transport as a pure turbulent diffusion flux and in the attempts sometimes to connect it to a turbulent diffusion coefficient, that for agreement with experimental data must be negative (counter-gradient, i. e. negative diffusion). We believe instead that this transport is controlled both by turbulence (turbulent diffusion) and by specific gasdynamics displacement of hot and cold volumes driven by pressure variation across the flame brush (in open flames the pressure is decreasing because of heat release). We will therefore use hereafter the term transport instead than turbulent diffusion expecially in the case of the counter-gradient phenomenon in order to enphasize the presence of an additional transport mechanism, usually prevailing in turbulent premixed flames, which is not directly associated with the turbulent pulsations of velocities.

It will be shown that the progress variable transport in open turbulent premixed flames has gradient character at the beginning of the flame, where turbulent diffusion prevails over the gasdynamics transport component (counter-gradient) because of the small flame brush width, and counter-gradient character at larger distance where the gasdynanamics effect starts to dominate. It is worth also mentioning that, if we create in a combusting turbulent premixed flow a nonuniform distribution of very small passive contaminants, there are strong grounds to believe that the nature of its transport would be of the gradient type controlled by the turbulent diffusion coefficient. This approach was in fact used in his experimental work by Prudnikov [15] where measurements of turbulent intensity and turbulent diffusion coefficient were performed using the optical diffusion method based on photo registration of the diffusion wake of luminous particles behind a point source placed in premixed flames. These experimental results did not show any "counter-gradient turbulent diffusion" at least at the back part of the flames where measurements were made.

In the TFC combustion model there is no contradiction between the experimentally observed increase of the flame brush width and the measured counter-gradient transport of the progress variable. The convective nature of the counter-gradient component does not directly affect in the model the flame brush thickness which, similarly to 
a non reactive mixing layer, is controlled by physical turbulent diffusion. In the present simulations of the counter-gradient phenomenon and its transition into gradient transport we have estimated the turbulent diffusion component using the standard $k-\epsilon$ model and the counter-gradient transport using a novel gasdynamics model developed here. These simulations demonstrate good agreement with the experimental data by Frank et al [3] in the transition from counter-gradient to gradient transport.

A similar idea was developed earlier in the paper [8] where high Reynolds number turbulent premixed combustion in a channel was simulated using the TFC model. In that case anyway, a very rough and less quantitatively accurate estimation of the gasdinamics transport component was used. This was based on the assumption of constant conditionally averaged velocities in the unburnt and burnt mixtures and gave the possible upper boundary for the counter-gradient transport component. It must be mentioned that conclusions consistent with this assumption followed also from the analysis of DNS results of turbulent premixed flames for the case of low turbulence levels $\left(u^{\prime} / s_{L}<1\right)$ performed by Veynante et al [12] when the two conditional velocities were found approximately constant across the flame brush and respectively equal to $s_{L}$ and $\Theta s_{L}$, where $\Theta=\rho_{u} / \rho_{b}$ is the ratio between the unburnt and burnt gases densities.

In our case of strong turbulence $\left(u^{\prime} / s_{L}>>1\right)$ such assumption is not valid. As it will be seen below in fact the assumption of constant conditional velocities across the turbulent flame brush results in a much larger effect. Comparing with experimental data available from Moss [1] for an open turbulent premixed flame, it is shown that the model developed in [8] overestimates the counter-gradient part of the total turbulent scalar flux by approximately three times.

In the present work a new improved model for the pressure-driven transport component is developed. This is based on the assumption of equal conditional average pressure $\overline{p_{u}}$ and $\overline{p_{b}}$ respectively in the unburnt and burnt fluid volumes. This model gives excellent agreement with Moss experimental data.

It is furthermore demonstrated that the capability to correctly model the pressuredriven transport component directly affects the accuracy with which we can estimate the heat release distribution. Consistently with the experimental observations made in $[11,16]$, it is shown, in fact, that the improved model for counter-gradient transport developed here yields an average heat release which is skewed towards value of the progress variable $\bar{c}>0.5$ with respect to the symmetric theoretical distribution of the Bray-Moss-Libby (BML) model (also predicted by our previous assumption of constant conditional velocities across the flame brush).

Experimental evidence supporting the present analysis is represented by the recent experimental data from [3]. According to these experiments in open premixed flames a transition from counter-gradient to gradient transport is observed at a given distance from the burner inlet when the ratio between the turbulent velocity fluctuation and the laminar flame speed $u^{\prime} / s_{L}$ increases, suggesting that physical turbulent diffusion becomes dominant over the gasdynamics pressure-driven mechanism. These experiments have been considered here for assessing the modelling idea proposed for the counter- 
gradient transport phenomenon.

\section{Kinematics equation. The counter-gradient transport phenomenon.}

The modelling kinematics equation for the Favre averaged progress variable in the case of flames in the ISP regime is given by:

$$
\bar{\rho} \frac{\partial \tilde{c}}{\partial t}+\bar{\rho} \tilde{u} \frac{\partial \tilde{c}}{\partial x}=\frac{\partial}{\partial x}\left(\bar{\rho} D_{t} \frac{\partial \tilde{c}}{\partial x}\right)+\rho_{u} U_{t}\left|\frac{\partial \tilde{c}}{\partial x}\right|
$$

where $\tilde{c}$ is the averaged progress variable $(\tilde{c}=0$ and $\tilde{c}=1$ correspond respectively to the cold reactants and hot products), $U_{t}$ is the turbulent combustion velocity, $D_{t}$ is the physical turbulent diffusion coefficient. The model source term in this equation determines propagation of the flame with velocity $U_{t}$ with respect to the unburnt fluid mixture while the gradient diffusion term determines the thickening of the turbulent flame brush according to the turbulent dispersion law.

This equation models the exact but unclosed equation:

$$
\bar{\rho} \frac{\partial \tilde{c}}{\partial t}+\bar{\rho} \tilde{u} \frac{\partial \tilde{c}}{\partial x}=\frac{\partial\left(-\bar{\rho} \widetilde{\left.u^{\prime \prime} c^{\prime \prime}\right)}\right.}{\partial x}+\bar{\rho} \widetilde{W}
$$

where $-\bar{\rho} \widetilde{u^{\prime \prime} c^{\prime \prime}}$ is the progress variable flux that as a rule is generally attributed a counter-gradient nature and $\bar{\rho} \widetilde{W}$ is the averaged rate of products formation. As we have mentioned in the introduction there is no contradiction between the gradient transport term in the model equation (1) and the as a rule counter-gradient transport term in the exact unclosed equation (2). This because the transport term in the model equation (1) contains only the turbulent diffusion component of the total transport term $-\bar{\rho} u^{\prime \prime} c^{\prime \prime}$, while the second gasdynamics convective component connected with the differential acceleration of hot and cold volumes in a nonuniform preassure field (the pressure-driven transport) is integrated together with the actual source term $\bar{\rho} \widetilde{W}$ in the model source term $\rho_{u} U_{t}|\partial \tilde{c} / \partial x|$ in equation (1). Obviously

$$
\rho_{u} \int_{-\infty}^{+\infty} U_{t}\left|\frac{\partial \tilde{c}}{\partial x}\right| d x=\int_{-\infty}^{+\infty} \bar{\rho} \widetilde{W} d x
$$

i. e. this procedure does not change the integral combustion intensity.

This interpretation of the model equation (1) was formulated by Zimont in [17] answering to a question from F. Williams about the possibility to predict countergradient transport within the framework of this model. The methodology for the analysis of the counter-gradient transport phenomenon based on this interpretation was subsequently developed in $[5,8]$ and now in this paper in more advanced form. It should also be remarked that Lipatnikov first realized in [18] that the interpretation of the turbulent diffusion term in the model equation (1) used as approximation of the transport term in unclosed equation (2) and the model source term as approximation of real source $\rho \widetilde{W}$ is non correct. A "joint closure" took place in fact while developing the model given by equation (1), such that the sum of the total transport term and the source term in (2) is instead approximated by the sum of turbulent diffusion and model 
source term in the model equation (1). At the same time Lipatnikov idea presented in [19] about the possibility to model counter-gradient transport is quite different from the one developed here.

It is significant to remember that equation (1) and the following equations (7) and (11) are valid only for flames with increasing flame brush width controlled by turbulent diffusion, i. e. in the case of flames in the ISP regime of combustion. In turbulent flames composed by laminar flamelets this regime takes place in the case of $u^{\prime}>>s_{L}$ and for time $t<<\tau_{t}\left(u^{\prime} / s_{L}\right)^{2}$ when turbulent transport by pulsation velocities $u^{\prime}$ prevails over the transport connected with flamelets local propagation with velocity $s_{L}$. In the case of turbulent flames composed by thickened flamelets at $R e_{t}>>1$ and $D a>>1$ with flamelet velocity $U_{f}>s_{L}$ this regime takes place for $t<\tau_{t} D a$ [8].

For larger times $t>\tau_{t}\left(u^{\prime} / s_{L}\right)^{2}$ (or $\left.t>\tau_{t} D a\right)$ turbulent premixed flames propagate according to the 1-D stationary propagating combustion front and instead of the model equation we can write the exact kinematics equation of the running wave (in coordinate system where the wave is motionless):

$$
\bar{\rho} \tilde{u} \frac{\partial \tilde{c}}{\partial x}=\rho_{u} U_{t}^{s t}\left|\frac{\partial \tilde{c}}{\partial x}\right|
$$

where in accordance with the ideas of Damköhler [20] and Shchelkin [21], in the case of strong turbulence we have $U_{t}^{s t} \approx u^{\prime}$, i.e. in the case of $u^{\prime}>s_{L}\left(\right.$ or $u^{\prime}>>U_{f}$ ) the turbulent combustion velocity does not depends on chemistry and molecular properties of the fuel/air mixture.

Comparison of equations (2) and (4) yields

$$
\rho_{u} U_{t}^{s t}\left|\frac{\partial \tilde{c}}{\partial x}\right|=\bar{\rho} \widetilde{W}-\frac{\left.\partial(\bar{\rho} u \bar{c})^{\prime \prime}\right)}{\partial x}
$$

demonstrating that the transport and source terms in the exact kinematical equation are clubbed under a single term given in our model equation (1) by $\rho_{u} U_{t}|d \tilde{c} / d x|$, i.e. they are intimately coupled for the 1-D stationary flame.

In the case of combustion occurring in the flamelet regime (and therefore with negligible probability to find burning mixture) the total transport term can be expressed as:

$$
-\bar{\rho} u^{\prime \prime} c^{\prime \prime}=-\bar{\rho} \tilde{c}(1-\tilde{c})\left(\bar{u}_{b}-\bar{u}_{u}\right)
$$

where $\bar{u}_{u}$ and $\bar{u}_{b}$ are conditioned averaged velocities of unburned and burned volumes. Simple estimation shows that for $\Theta>>1$ (strong heat release) and $\delta_{t}^{s t}>>L$, the ratio of the turbulent diffusion flux to the pressure-driven flux is $\sim\left(u^{\prime} L / \delta_{t}^{s t}\right) /\left(U_{t}^{s t} \Theta\right)<<1$, i. e. the counter-gradient transport component and the actual source term are clubbed under the single term $\rho_{u} U_{t} \partial \tilde{c} / \partial x$. In the case of the ISP combustion regime where flames have increasing flame brush width and $\delta_{t}<\delta_{t}^{s t}$ we have extracted the turbulent diffusion component (controlling in our model the brush width) into a separate term and left the remaining gasdynamics transport and the chemical source clubbed into the model source term. 
According to these ideas which were originally proposed in $[8,5]$, the progress variable flux can be split into two contributions: one of gradient nature generated by real turbulent diffusion transport which has the property to increase the flame brush thickness (according to the turbulent dispersion law) and the second of counter-gradient nature which is of convective type and is generated by the pressure drop across the turbulent flame brush. The first component of the transport is controlled by positive turbulent diffusion coefficient that can be estimated using usual turbulence models and the second component can be estimated from some gasnamical model that is strongly connected with the nonuniform averaged pressure distribution (in our case of open flames, with the averaged pressure drop across the flame and resulting in the countergradient gasdynamical component).

The possibility of such decomposition can be better understood observing the exact expression for the progress variable flux (6). This expression shows that the turbulent scalar flux $-\bar{\rho} u^{\prime \prime} c^{\prime \prime}$ has gradient (counter-gradient) nature when $\bar{u}_{b}<\bar{u}_{u}\left(\bar{u}_{b}>\bar{u}_{u}\right)$. In absence of a negative pressure gradient which can accelerate the products more than the reactants, the effect of turbulent dispersion will be to generate penetration of burnt mixture inside the unburnt one at the cold boundary and penetration of unburnt mixture inside the burnt one at the hot boundary. According to this description the conditional velocities will be such that $\bar{u}_{b}<\bar{u}_{u}$. In presence of a pressure drop across the turbulent flame brush, the light burnt gases are accelerated more than the unburnt ones. This reduces the gradient transport or even transform it into the counter-gradient phenomenon when $\bar{u}_{b}>\bar{u}_{u}$.

The connection of the counter-gradient transport with the averaged pressure gradient is well known. In recent DNS work, it has been shown a well-defined effect of pressure pulsations [22]. This effect is connected with the instantaneous difference of conditional averaged pressures of unburned $\bar{p}_{u}$ and burned gases $\bar{p}_{b}$ as the boundary between these gases is a moving flamelets that gives $\bar{p}_{u}>\bar{p}_{b}$. Therefore, for example, even at $\bar{p}=$ const there is some counter-gradient component. In our simulations below we ignore this effect.

It must be observed that, because of the convective nature of the pressure-driven transport, while developing the TFC combustion model we have assumed that it doesn't have the capability to change the thickness of the flame boundary layer, a property which is typical of turbulent diffusion phenomena.

Subtracting now equation (1) from (2) we obtain the following relation:

$$
\rho_{u} U_{t}\left|\frac{\partial \tilde{c}}{\partial x}\right|=\bar{\rho} \widetilde{W}+\frac{\partial}{\partial x}\left[-\bar{\rho} \widetilde{u^{\prime \prime} c^{\prime \prime}}-\bar{\rho} D_{t} \frac{\partial \tilde{c}}{\partial x}\right]
$$

which formally shows that the model source term in (1) accounts at the same time for real heat release and the pressure-driven (counter-gradient in our case of open premixed flames) transport component. In order to extract the counter-gradient transport term from (7) two approaches may be used: a) give a model for the average heat release $\bar{W}$, b) model directly the counter-gradient transport component (in this case relation (7) will give an expression for $\bar{\rho} \widetilde{W}$ ). 
In [8] approach a) has been followed. It was assumed that the actual averaged chenical source term in equation (2) is proportional to the probability to find the flamelet at a given position $p_{f}(x)$; this probability is related to the probability of finding products $P_{b}(x)$ at the given position by the relation:

$$
P_{b}(x)=\int_{-\infty}^{x} p_{f}(\xi) d \xi \Rightarrow p_{f}(x)=\frac{\partial P_{b}(x)}{\partial x}
$$

Note also the following expression for the averaged progress variable (as flamelets are thin, the probability to have $0<c<1$ has been neglected):

$$
\bar{c}=P_{b} 1+\left(1-P_{b}\right) 0=P_{b}
$$

Therefore we can write:

$$
\bar{\rho} \widetilde{W}=\text { const }\left|\frac{\partial \bar{c}}{\partial x}\right|
$$

where the constant is equal to $\rho_{u} U_{t}$ as can be shown by integrating equation (7) from $-\infty$ to $+\infty$.

Using (10) in (7) the following expression for the second order velocity-progress variable correlation is obtained (where it is assumed $d \bar{c} / d x>0$ without loss of generality):

$$
\frac{\partial\left(-\bar{\rho} \widetilde{u}^{\prime \prime} c^{\prime \prime}\right)}{\partial x}=\rho_{u} U_{t} \frac{\partial}{\partial x}(\tilde{c}-\bar{c})+\frac{\partial}{\partial x}\left[\bar{\rho} D_{t} \frac{\partial \tilde{c}}{\partial x}\right]
$$

which integrated from $-\infty$ to $x$ yields:

$$
-\bar{\rho} u^{\prime \prime} c^{\prime \prime}=\rho_{u} U_{t}(\tilde{c}-\bar{c})+\bar{\rho} D_{t} \frac{\partial \tilde{c}}{\partial x}
$$

This relation explicitely gives evidence that the second order Favre correlation between the progress variable and velocity fluctuations is composed of two contributions:

a) real turbulent transport (modelled here with an eddy diffusivity assumption) which is responsible for the thickening of the ISP flame brush;

b) a contribution which is proportional to the integral of the difference between the model $\rho_{u} U_{t} d \tilde{c} / d x$ and the real chemical source term $\bar{\rho} \widetilde{W}=\rho_{u} U_{t} d \bar{c} / d x$. This contribution can be expressed with simple algebraic manipulations as:

$$
\rho_{u} U_{t}(\tilde{c}-\bar{c})=-\rho_{u} U_{t} \tilde{c}(1-\tilde{c}) \frac{\Theta-1}{1+\tilde{c}(\Theta-1)}
$$

It should be again enphasized that the point of view adopted here is that the turbulent flame brush increases for the effect of turbulent dispersion, independently on the nature of the progress variable flux (of gradient or counter-gradient type). Nevertheless it sometimes stated that in case of a total scalar flux of counter-gradient type the flame brush becomes thinner whereas in case of gradient type it becomes thicker [12]. We think that this point of view is obtained as result of interpreting the progress variable flux in turbulent premixed flames only as a turbulent diffusion flux of passive concentration. The term "counter-gradient turbulent diffusion" is in fact often 
associated with the phenomenon of counter-gradient transport which, we stress here, is not connected with a negative value for the turbulent diffusion coefficient.

We believe that in open flames a decreasing flame brush width is highly improbable. Numerous experiments show in fact that the flame brush width increases with distance along the flame similarly to a diffusing wake, despite of the commonly observed countergradient nature of the total transport. In principle this thickness increases until a "statistically steady" finite value which is unattainable in practical combustion devices. At the same time, in presence of strong external pressure gradient (combustion in a nozzle, for example) the qualitative picture could be more complicated, but we do not analize this case here.

As already mentioned the nature of the progress variable transport (gradient or counter-gradient) can be determined by analysing the difference between the average conditional velocities $\bar{u}_{u}$ and $\bar{u}_{b}$ respectively in the cold and hot gases as shown by relation (6). Solving the system of equations given by (6), (12) and the mass conservation equation in the two unknown $\bar{u}_{u}$ and $\bar{u}_{b}$ :

$$
\begin{aligned}
-\bar{\rho} \tilde{c}(1-\tilde{c})\left(\bar{u}_{b}-\bar{u}_{u}\right) & =\rho_{u} U_{t}(\tilde{c}-\bar{c})+\bar{\rho} D_{t} \frac{\partial \tilde{c}}{\partial x} \\
(1-\tilde{c}) \bar{u}_{u}+\tilde{c} \bar{u}_{b} & =[1+(\Theta-1) \tilde{c}] U_{t}
\end{aligned}
$$

we obtain the following expression for the conditional velocities:

$$
\begin{aligned}
& \bar{u}_{u}=\frac{D_{t}}{1-\tilde{c}} \frac{d \tilde{c}}{d n}+U_{t} \\
& \bar{u}_{b}=-\frac{D_{t}}{\tilde{c}} \frac{d \tilde{c}}{d n}+\Theta U_{t}
\end{aligned}
$$

These relations show that each of the conditional velocities is composed by two contributions: one related to turbulent dispersion and the other to convection.

In the case of a steady flame ( $t \rightarrow \infty$, constant thickness and velocity) the flame is described by the equation (4), i.e. in (16) and (17) we must put $D_{t}=0$, resulting in the two conditional velocities being constant across the flame brush, $\bar{u}_{u}=\bar{u}(-\infty)=U_{t}^{s t}$ and $\bar{u}_{b}=\bar{u}(+\infty)=\Theta U_{t}^{s t}$. This also implies constant conditional pressures $\left(\bar{p}_{u}=\bar{p}(-\infty)=\right.$ const, $\bar{p}_{b}=\bar{p}(+\infty)=$ const) as it will be shown in the next paragraph. Such value of velocities correspond in fact to the assumption that cold and hot volumes move inside the flame without mutual force interaction. Obviously this strongly overestimates the counter-gradient transport (for tipical conditions approximately three times, see below) and corresponds to the possible upper boundary of this phenomenon. We will call this model "the rough model" or "the upper estimation" model.

More realistic modelling must take into account this interaction, i. e. the acceleration of relatively slow cold volumes by more fast hot volumes and viceversa. Such model is developed in the next section and we will call it "the accurate model" or "the gasdynamics model". 


\section{A gasdynamics model for the counter-gradient transport in open flames}

In this section we analyze 1-D stationary premixed flames. These results will then be applied to open flames with increasing width assumiing that the pressure gradient in this case is the same as in 1-D stationary flames. The good agreement between numerical simulations and experimental data confirm the validity of this hypothesis at least in first approximation.

According to the idea presented in section 2, the progress variable transport in open turbulent premixed flames is composed by two fundamental contributions: real gradient turbulent diffusion which is responsible for the growth of flame brush thickness and counter-gradient transport related to the pressure drop across the flame brush due to heat release. In this section we will present a gasdynamics model for the pressure driven component of the progress variable transport in the case of 1-D stationary flames which will be applied to real open flames with increasing flame brush width.

The basis for this analysis is represented by equations (4)-(6). For estimating the pressure-driven component we assume here that the conditional velocities in (6) are controlled only by gasdynamics, i. e. we ignore the effect of turbulent dispersion. In this case the term $-\bar{\rho} \widetilde{u^{\prime \prime} c^{\prime \prime}}$ includes only the counter-gradient transport component, and in fact the model source (5) of equation (4) contains the actual source term and the pressure-driven counter-gradient component. The results obtained under these conditions can therefore be extended to ISP flames by using equation (1). In this equation in fact the gradient transport term is controlled by turbulent diffusion and the model source term contains the actual source term and the pressure-driven countergradient component.

Consider therefore the conservation equations for mass, momentum and reactants total pressure (iso-entropic condition for reactants). The equations have been written here for $U_{t}^{s t}=1 \mathrm{~m} / \mathrm{s}$ and $\rho_{u}=1 \mathrm{~kg} / \mathrm{m}^{3}$ (or equivalently in normalised form):

$$
\begin{aligned}
(1-\bar{c}) \bar{u}_{u}+\bar{c} \frac{\bar{u}_{b}}{\Theta} & =1 \\
\bar{p}+(1-\bar{c})\left[\bar{u}_{u}^{2}+\overline{u_{u}^{\prime 2}}\right]+\frac{1}{\Theta} \bar{c}\left[\bar{u}_{b}^{2}+\overline{u_{b}^{\prime 2}}\right] & =p_{-\infty}+1+\overline{u^{\prime 2}}-\infty \\
\bar{p}_{u}+\frac{1}{2}\left[\bar{u}_{u}^{2}+\overline{u_{u}^{\prime 2}}\right] & =p_{-\infty}+\frac{1}{2}\left(1+\overline{u^{\prime 2}}-\infty\right)
\end{aligned}
$$

where the average pressure $\bar{p}$ is given by $\bar{p}=(1-\bar{c}) \bar{p}_{u}+\bar{c} \bar{p}_{b}$. In the case introduced in the previous paragraph we have seen that $\bar{\rho} \widetilde{W}=\rho_{u} U_{t} d \bar{c} / d x$ implies uniform conditional velocities across the turbulent flame brush and equal to $\bar{u}_{u}=1$ and $\bar{u}_{b}=\Theta$ in the present case. In this case equation (18) becomes redundant and the system therefore reduces to two equation in the two conditional pressures as unknowns.

If we subtract equation (20) from equation (19) and require the fluctuating terms to cancel, we end up with the following two relations:

$$
\bar{c}\left(\bar{p}_{b}-\bar{p}_{u}\right)+\left(\frac{1}{2}-\bar{c}\right) \bar{u}_{u}^{2}+\frac{1}{\Theta} \bar{c} \bar{u}_{b}^{2}=\frac{1}{2}
$$




$$
\left(\frac{1}{2}-\bar{c}\right){\overline{u_{u}^{\prime}}}^{2}+\frac{1}{\Theta} \bar{c}{\overline{u_{b}^{\prime}}}^{2}=\frac{1}{2}{\overline{u_{u}^{\prime 2}}}_{-\infty}
$$

The second of these relations gives a turbulent velocity fluctuation $\overline{u_{b}^{\prime 2}}+\infty$ at the hot boundary which is equal to $\Theta / 2 \overline{u_{u}^{\prime 2}}$. This result is consistent with several experimental works where the turbulent velocity fluctuation has been found increasing across the turbulent flame brush. For example, Moreau [23] has found in oblique high velocity planar methane/air flames at equivalence ratio 0.8 that the axial turbulent fluctuating velocity increases inside the flame, reaching a value about three times that measured in the cold flow.

In the particular case of $\bar{u}_{u}=1$ and $\bar{u}_{b}=\Theta$ analysed in the previous section we have $\bar{p}_{u}-\bar{p}_{b}=(\Theta-1)$. Obviously this is an hypothetical unrealistic case. We will instead analyse here the case where $\overline{p_{u}}=\overline{p_{b}}=\bar{p}$ ignoring the small difference connected with the movement of the boundary between them as we already mentioned above. The idea behind this assumption is that strong interaction between hot and cold volumes is responsible for the local homogeneization of conditional pressures.

Under this assumption we have from equation (21):

$$
\left(\frac{1}{2}-\bar{c}\right) \bar{u}_{u}^{2}+\frac{1}{\Theta} \bar{c} \bar{u}_{b}^{2}=\frac{1}{2}
$$

The system given by (18) and (23) can be solved with simple algebraic manipulations. This yields the following expressions for $\bar{u}_{u}$ and $\bar{u}_{b}$ :

$$
\begin{aligned}
& \bar{u}_{b}=\frac{-\beta+\sqrt{-4 \alpha \gamma+\beta^{2}}}{2 \alpha}, \quad \bar{u}_{u}=\frac{1-\bar{u}_{b} \bar{c} / \Theta}{1-\bar{c}} \\
& \alpha=\frac{\bar{c}}{\Theta}\left[\frac{0.5-\bar{c}}{(1-\bar{c})^{2}} \frac{\bar{c}}{\Theta}+1\right], \quad \beta=-2 \frac{\bar{c}}{\Theta} \frac{0.5-\bar{c}}{(1-\bar{c})^{2}}, \quad \gamma=\frac{0.5-\bar{c}}{(1-\bar{c})^{2}}-0.5
\end{aligned}
$$

The expression obtained now for the conditional velocities gives the opportunity to calculate the average heat release within the turbulent flame brush using equation (7). We will assume $\partial \bar{c} / \partial x$ to be distributed as a Gaussian function (validation of this hypothesis has been performed for example in [9] in the case of simple laboratory two-dimensional turbulent premixed flames):

$$
\frac{\partial \bar{c}}{\partial x}=\frac{1}{\sqrt{2 \pi \sigma^{2}}} \mathrm{e}^{-(x-a(t))^{2} / 2 \sigma^{2}}
$$

where $a(t)$ is the position of $\bar{c}=0.5$ and $\sigma$ the standard deviation the Gaussian function (related to the flame brush thickness). Introducing the non-dimensional spatial variable $\xi=(x-a(t)) / \sqrt{2 \sigma^{2}}$, the average heat release non-dimensionalised with $\rho_{u} U_{t} / \sqrt{2 \sigma^{2}}$ is given by:

$$
\frac{\bar{\rho} \widetilde{W}}{\rho_{u} U_{t} / \sqrt{2 \sigma^{2}}}=\frac{1}{\sqrt{\pi}} \mathrm{e}^{-\xi^{2}} \frac{\partial}{\partial \bar{c}}\left[\tilde{c}-\bar{c}(1-\tilde{c}) \frac{\bar{u}_{b}-\bar{u}_{u}}{U_{t}}\right]
$$

In the hypothetical case of conditional velocities constant across the flame brush, this expression yields the result already introduced at paragraph, i.e. $\bar{\rho} \widetilde{W}=\rho_{u} U_{t}|\partial \bar{c} / \partial x|$. Figure 1 shows the distribution of the conditional velocities non-dimensionalised with $U_{t}$ and the distribution of the non-dimensional heat release, $\partial \bar{c} / \partial \xi$ and $\partial \tilde{c} / \partial \xi$. The 

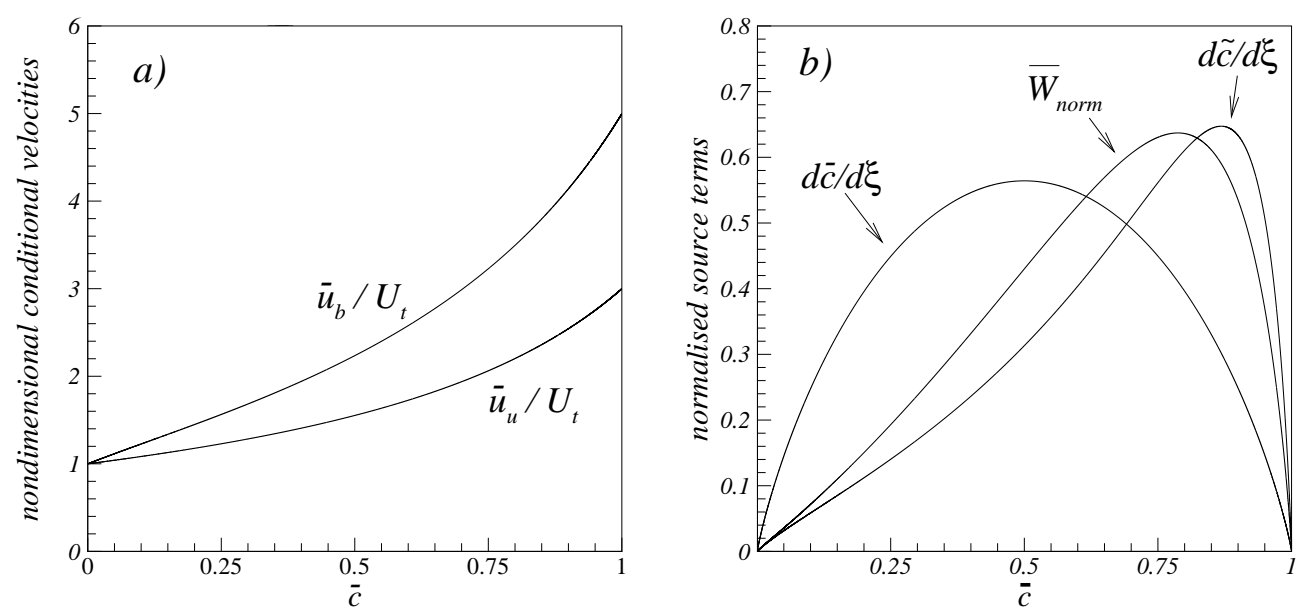

Figure 1. Distribution of a) non-dimensional conditional velocities and b) normalised progress variable source terms across the flame brush. Gaussian distribution assumed for $d \bar{c} / d x .\left(\rho_{u} / \rho_{b}=5\right)$.

heat release is shifted toward the front part of flame brush with respect to the model source term, the maximum shift obtained for the model based on uniform distribution of conditional pressures and velocities across the turbulent flame brush (when $\bar{u}_{b}-\bar{u}_{u}$ attains its maximum value). In the case of the model obtained assuming $\bar{p}_{u}=\bar{p}_{b}$ the heat release $\bar{W}_{\text {norm }}$ is less shifted toward the front part of the flame brush.

It is interesting to note that such situation has been observed experimentally in [11] and [16] where a large dataset of experimental data on turbulent premixed flame of various types (v-, conical, stagnation and swirl stabilised flames) was analyzed. The experiments from $[11,16]$ will be considered in paragraph 5 for a qualitative validation of the result obtained with the present model.

\section{Modelling of the turbulent flame speed}

The turbulent flame speed $U_{t}$ of a 1-D flame is given by the product between the local propagation velocity $U_{f}$ and the surface area for unit of flame cross sectional area $\left(\bar{S} / S_{0}\right)$ of the thin flamelet, i. e. $U_{t}=U_{f}\left(\bar{S} / S_{0}\right)$. These two quantities have been estimated by asymptotics analysis at large $R e_{t}$ and $D a$ numbers based on the Kolmogorov methodology: the equilibrium fine-scale turbulence from the inertial interval controls the thickened flamelet combustion velocity giving $U_{f}>s_{L}$ and flamelet width $\delta_{f}>\delta_{L}$, the equilibrium small scale flame wrinkles controls the practically constant flame surface area and finally nonequilibrium large-scale wrinkles control the flame brush width which increases similarly to a non-reactive mixing layer $[4,8]$.

In this model the microturbulent transport of heat or concentrations $\left(\chi_{f} \simeq D_{f}\right)$ within the flamelets thickness is discribed by the well-known Richardson diffusion law $\chi_{f} \simeq \epsilon^{1 / 3} \delta_{f}^{4 / 3}$ where $\epsilon$ is the averaged rate of turbulent kinetic energy dissipation. The 
following expressions have been obtained:

$$
\begin{aligned}
& U_{f} \simeq u^{\prime} D a^{-1 / 2}, \delta_{f} \simeq L D a^{-3 / 2}, \chi_{f} \simeq D_{t} D a^{-2} \Rightarrow \\
& \left(\bar{S} / S_{0}\right) \simeq D a^{3 / 4} \simeq\left(u^{\prime} / U_{f}\right)^{3 / 2} \approx\left(L / \delta_{f}\right)^{1 / 2} .
\end{aligned}
$$

According to the first of these relations, the local propagation velocity of the thickened flamelet increases with decreasing Damköhler number (for example, reducing of the chemistry rate). This can be explained considering that a reduction in Damköhler number produces an increase in the flamelet thickness (the second relation). It results therefore an increase in the micro-turbulent transport coefficient (the third relation) because of small vortices in the inertial range falling inside the flamelet. This is dominant over the decreasing effect produced by the reduction in chemistry rate and generates an overall increase in the local propagation velocity. The fourth expression shows that there is connection between the flamelet area and the flamelet velocity: an increase in the flamelet velocity (or an increase in the flamelet width) produces a decrease in the flamelet sheet area. The physical reason of this effect is self-smoothing of the wrinkled sheet by its movement (or by the increase in the flamelet width): an increase in $U_{f}$ (or decreas in $\delta_{f}$ ) "comsumes" the smallest existing wrinkles and it decreases the flame surface area. This self-compensation mechanism is responsible for the relatively weak chemistry dependence of $U_{t}$ shown by experiments.

The final expression for the turbulent combustion velocity is as follows:

$$
U_{t}=U_{f}\left(\bar{S} / S_{0}\right)=A u^{\prime} D a^{1 / 4}=A u^{3 / 4} s_{L}^{1 / 2} \chi^{-1 / 4} L^{1 / 4},
$$

where $A \sim 1$ is an empirical coefficient, $\chi$ is the molecular heat transfer coefficient (referred to the unburned mixture in the simulations, i. e. $\chi=\chi_{u}$ ). It is worth emphasizing that all these expressions were derived from the physical model and, with the exception of the coefficient $A$, they do not contain any quantitative empirical parameter. From (29) we can see that the turbulent flame speed depends from chemistry according to $U_{t} \sim \tau_{c h}^{-1 / 4}$ in a much weaker way than the case of laminar combustion where $s_{L} \sim \tau_{c h}^{-1 / 2}$. We expect that in the case of laminar flamelets, the chemistry dependence for $U_{t}$ would be also weaker due to self-compensation mechanism mentioned above but for laminar flamelets similar formulas do no exist. It seems obvious that this self-compensation mechanism is responsible for many quantitative paculiarities of turbulent combustion occurring according to the flamelet mechanism and in particular the relatively weak dependence of $U_{t}$ on chemistry.

As mentioned in the introduction there is direct experimental evidence of the existence of thickened flamelets.

Bradley et al [24] have correlated many experimental results for premixed turbulent flames in order to obtain an expression for the turbulent flame speed as function of the relevant physico-chemical parameters. This expression, assuming that all molecular transport coefficients are equal, is given by:

$$
U_{t} \simeq u^{\prime} D a^{0.3} R e_{t}^{-0.15}
$$


This empirical expression is close to the theoretical one (29). The weaker dependence of $U_{t}$ on $u^{\prime}$ in relation (30) was connected in [18] wih the effect of the flamelet stretch which is present in (30) and not in (29). When the strain rate becomes large, local flamelet quenching may occur. This is not taken in account in the theoretical expression (29) which is therefore valid if the local strain rate is sufficiently small. The effect of large strain rates is to reduce the local flamelets velocities and even cause their extinction. This effect has been incorporated [25] in the expression for $U_{t}$ using a model developed by Bray [26] for the stretch-effect. According to this model, a stretch factor $G$ which represent the probability for the instantaneous turbulent kinetic energy dissipation rate $\epsilon$ to be less than the critical extinction value $\epsilon_{c r}$ has been introduced. Assuming a log-normal distribution for $\epsilon$ [26], the following expression for the stretching factor is obtained:

$$
G=0.5 \operatorname{erfc}\left[-\frac{1}{\sqrt{2 \sigma}}\left(\ln \left(\epsilon_{c r} / \epsilon\right)+\frac{\sigma}{2}\right)\right]
$$

where erfc denotes the complementary error function, $\sigma=\mu \ln (L / \eta)$ the standard deviation ( $\mu=0.28$ being a constant). The final expression for $U_{t}$ is therefore given by:

$$
U_{t}=A G u^{\prime} D a^{1 / 4}
$$

It has to be observed that an accurate estimation of $\epsilon_{c r}$ is necessary to correctly account for the "bending" of $U_{t}$ in the dependence $U_{t}=f\left(u^{\prime}\right)$; a reduction in $U_{t}$ results in fact at large turbulent intensities.

Validation of the combustion model based on the expression (32) for the turbulent flame speed and for the bending effect can be found in [18, 27]. Application of the model to industrial premixed combustion can be found in [10] and validation for the case of a high speed 2D turbulent premixed flame in [8].

\section{Validation}

The modelling ideas proposed here to account for transport of reactive components in turbulent premixed flames have been validated with three sets of experimental data.

The first of these consists in the experiments performed by Moss [1] for an open LPG/air turbulent premixed flame at stoichiometric conditions. These data correspond therefore to a situation that minimizes $u^{\prime} / s_{L}$ for a given value of $u^{\prime}$, i.e. such to give a small gradient transport contribution. This favours the validation of the model developed here for the counter-gradient component of the total scalar transport.

The second part of the validation is presented here only on a qualitative basis in order to show that the present model for counter-gradient transport gives a heat release distribution across the flame brush which is consistent with available experimental observations. The experimental data considered for this validation are from Cheng and Sheperd [11] and Cheng [16] who have analysed turbulent premixed flames in various configurations. 


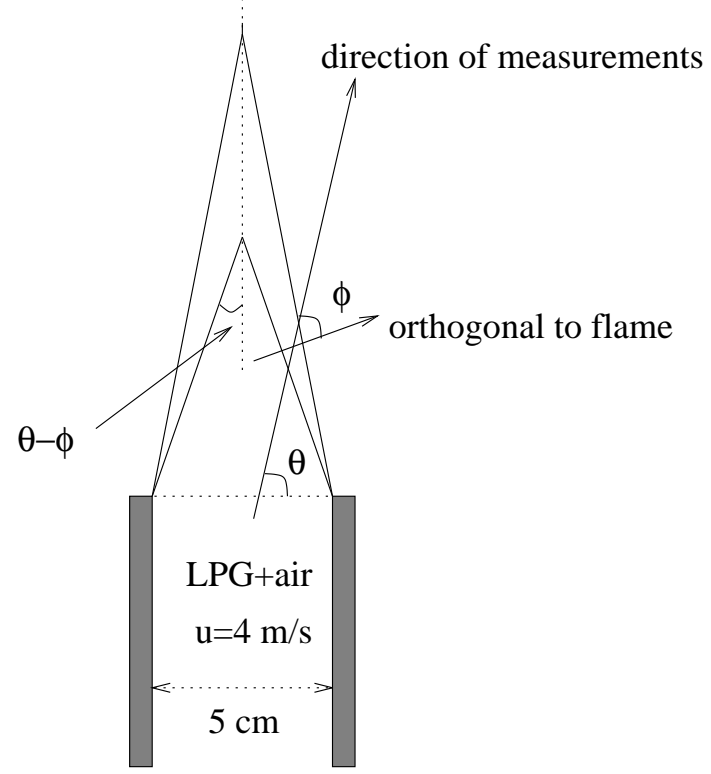

a)

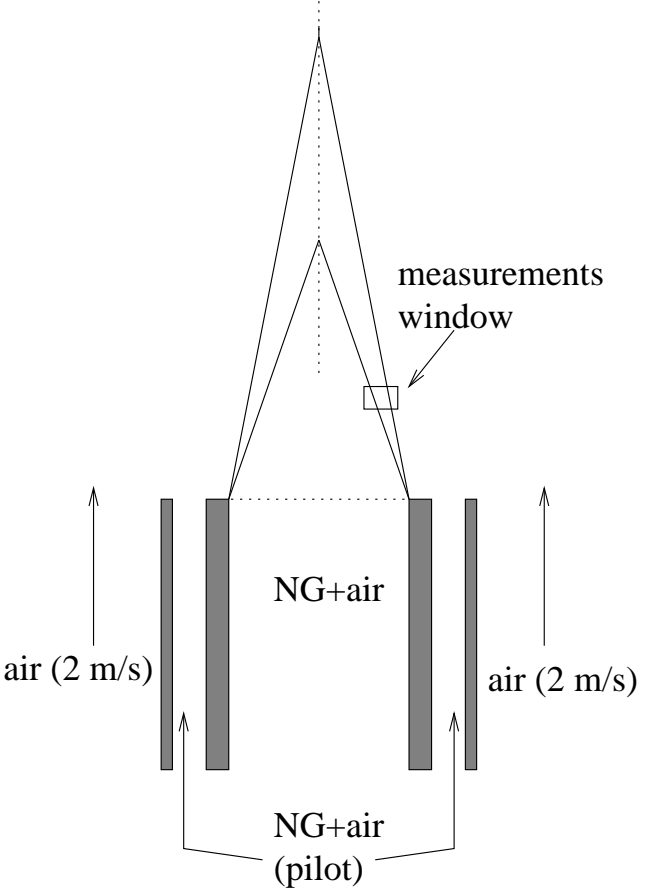

b)

Figure 2. Schematics of combustor in [1] experiments (left) and [3] experiments (right).

The third set of data consists in the experiments recently perfomed by Frank et al [3] for an axisymmetric natural gas/air open turbulent premixed flame stabilised in a co-flowing air stream by a pilot jet of hot gases. In this case, the conditional average velocities $\bar{u}_{u}$ and $\bar{u}_{b}$ have been measured for different values of the ratio $u^{\prime} / s_{L}$ (mainly by changing the fuel/air mixture stoichiometry) and a transition from gradient to countergradient transport has been observed for decreasing of $u^{\prime} / s_{L}$. These data are therefore of particular interest for the overall validation of the model for the total scalar flux developed here.

It must be stressed that the model developed here for countergradient transport refers to 1-D stationary flames. Its application to real flame with increasing flame brush width is therefore an approximation. We believe anyway that this approximation is reasonable for open flames as these flames have simple structure and in most of them the dominating part of the transport is the countergradient component. In the case instead of real confined flames with complex structure and where the pressure gradient is determined also by other factors such approach is not applicable. The development of a more general model able to account for countergradient transport also in these flame will be developed in future. 


\subsection{Validation with Moss experiments [1] for an open turbulent premixed flame}

The schematics of the experiments for this open turbulent premixed flame is shown in figure $2 \mathrm{a}$ ). The burner is composed by a pipe, $5 \mathrm{~cm}$ in diameter and $60 \mathrm{~cm}$ in length. The pipe is fed with air and LPG (approximately 70 percent propane and 30 percent propene) which becomes fully premixed at the pipe exit. The velocity at the burner exit is $\bar{u} \simeq 4 \mathrm{~m} / \mathrm{s}$, approximately ten times the laminar flame speed. The velocity $\mathrm{rms} \sqrt{\overline{u^{\prime 2}}}$ at the center of the pipe exit is $0.5 \mathrm{~m} / \mathrm{s}$.

Measurements have been taken across the flame brush along a line inclined $23^{\circ}$ with the pipe axis. The angle observed between the flame and the burner axis was approximately $\theta-\phi=16^{\circ}$. Concentration (the progress variable) was measured by light scatter technique and velocities by laser Doppler velocimetry. Moss experimental data have been subsequently postprocessed in [13]; the data reported in this last reference have been adopted here for validation.

A full CFD analysis of this case hasn't been performed. Using the available information, we have estimated that the order of magnitude of the turbulent diffusion component with the total scalar flux (the gradient component of transport) is small in comparison to the counter-gradient component. For these experiments we have in fact a turbulent flame speed of the order of $U_{t} \sim \bar{u} \sin (\theta-\phi)=4 \sin (16)=1.1 \mathrm{~m} / \mathrm{s}$. Assuming the integral turbulent length scale at the pipe exit to be in the range of $L \simeq 1.5 \mathrm{~mm}$ we have $\epsilon \simeq 0.37 \overline{u^{\prime 3}} / L=31 \mathrm{~m}^{2} / \mathrm{s}^{3}$ and the turbulent diffusion coefficient $D_{t} \simeq 0.09\left(3 / 2 \overline{u^{\prime 2}}\right)^{2} / \epsilon=0.0004 \mathrm{~m}^{2} / \mathrm{s}$. The flame brush increases in thickness according to the turbulent dispersion law, i.e. $\delta_{f} \simeq \sqrt{2 D_{t} H / \bar{u}}$ where $H$ is the height from the pipe exit at which measurements have been taken, i.e. $H \simeq 0.0351 \mathrm{~m}$ (assuming the flame to be flat). We have therefore $\delta_{f} \simeq \sqrt{2 \times 0.0004 \times 0.0351 / 4}=0.00265 \mathrm{~m}$ and assuming the maximum progress variable gradient to be approximately $1 / \delta_{f}=1.0 / 0.00265 \mathrm{~m}^{-1}$ at $\tilde{c}=0.5$, we have a contribution to $\bar{u}_{u} / U_{t}$ and $\bar{u}_{b} / U_{t}$ from turbulent dispersion roughly equal to $0.0008 / 0.0053=0.27 \mathrm{~m} / \mathrm{s}$. The experimental values of $\bar{u}_{b} / U_{t}$ and $\bar{u}_{u} / U_{t}$ at this location are respectively equal to $\simeq 6$ and $\simeq 3$, i.e. the contribution to the conditional velocities by real turbulent transport is small compared to the contribution by countergradient transport.

We compare therefore here only the contribution to the transport generated by the pressure drop across the turbulent flame brush.

Figure 3 shows the non-dimensional turbulent scalar flux $\bar{\rho} \widetilde{u^{\prime \prime} c^{\prime \prime}} / \rho_{u} U_{t}$ and the nondimensional conditional velocities $\bar{u}_{u} / U_{t}$ and $\bar{u}_{b} / U_{t}$ calculated using the two models presented in this work. The model based on the assumption of uniform conditional velocities across the flame brush, results in an overestimation of the experimental progress variable of about three times. The model based on the assumption $\overline{p_{u}}=\overline{p_{b}}$ gives instead very good agreement with the experimentally determined progress variable flux, the small overprediction probably related to having neglected here the opposite effect of gradient diffusion. The conditional velocities calculated with this model are also in good agreement with the experimental data as shown in figure $3 \mathrm{~b}$ ). Note that the predicted 

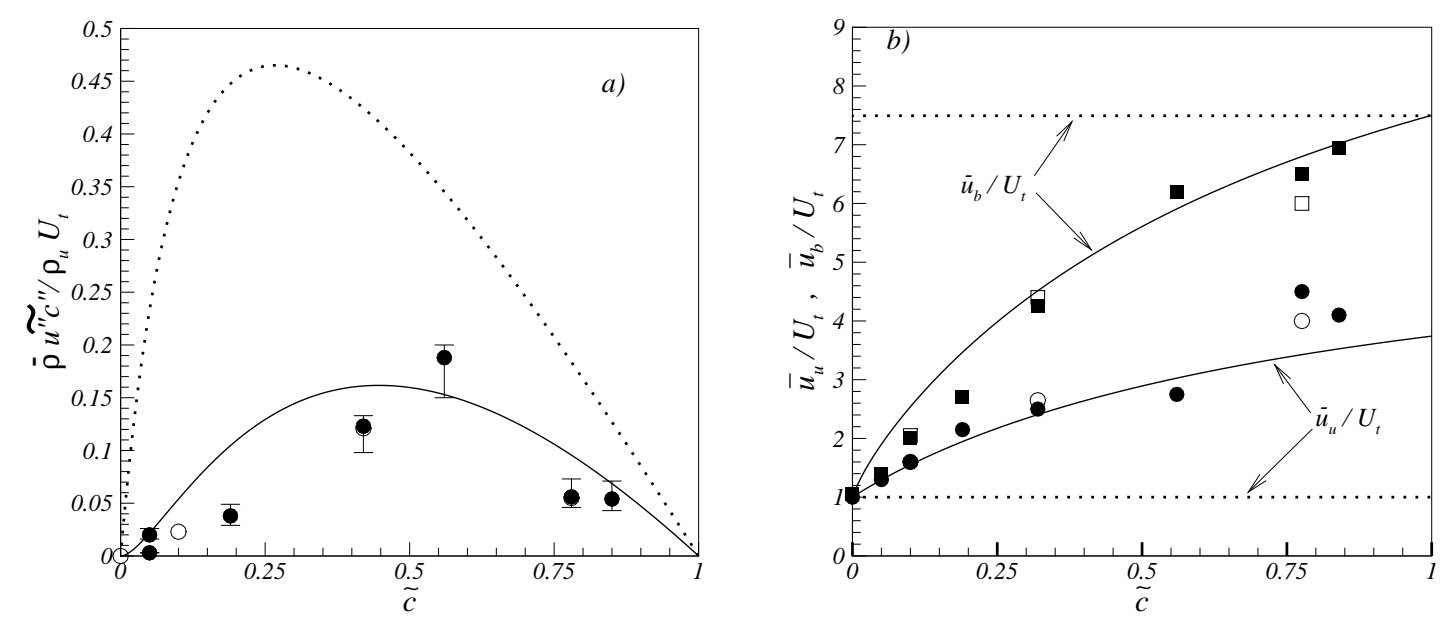

Figure 3. Comparison with a) progress variable flux and b) conditional velocities from Moss experiments. Simulations: ...... results from the rough (upper estimation of $\bar{\rho} \widehat{u^{\prime \prime} c^{\prime \prime}}$ model), —— results from the accurate (gasdynamics) model. Experiments: a) calculated in [13] from Moss measured conditional mean velocities, $O$ calculated in [13] from Moss measured $\bar{\rho} \widehat{u^{\prime \prime} c^{\prime \prime}}$. b) $\bullet$ measured experimental $u_{u}, \bigcirc u_{u}$ from measured $\bar{\rho} u^{\prime \prime} c^{\prime \prime}$, measured experimental $u_{b}, \square u_{b}$ from measured $\bar{\rho} \widehat{u^{\prime \prime} c^{\prime \prime}}$

$\bar{u}_{u}$ and $\bar{u}_{b}$ are on average respectively slightly smaller and larger than the corresponding measured values which might be again the consequence of having neglected the effect of gradient transport (which increases $\bar{u}_{u}$ and decreases $\bar{u}_{b}$ ).

\subsection{Qualitative validation of heat release distribution with Cheng experimental data}

Cheng et al $[11,16]$ have studied turbulent premixed flames in various configurations, including impinging, swirl stabilised and v-flames. During these experiments the average heat release distribution across the flame brush via the measurement of the flamelets crossings frequency $\nu(x)$ has been determined. In the flamelet regime in fact the average heat release can be expressed as [28]:

$$
\widetilde{\rho} \widetilde{W}=\rho_{u} s_{L} \frac{\nu(x)}{U_{n}(x)}
$$

where $\nu(x)$ is the flamelet crossing frequency (average number of flamelet crossing for unit of time) and $U_{n}(x)$ the mean flamelet crossing speed within the turbulent flame in a laboratory frame. The experimental results show that the flamelet crossing frequency is skewed toward values of $\bar{c}>0.5$ with respect to the symmetrical heat release distribution predicted by the BML theory and proportional to $\bar{c}(1-\bar{c})$. Under the assumption of constant $U_{n}(x)$ and laminar flame speed $s_{L}$, this indicates that also the averaged heat release is skewed in a similar fashion. The model for counter-gradient trasport based on $\bar{p}_{u}=\bar{p}_{b}$ has been applied here to the case of a turbulent premixed flame with $\theta=3$ to emulate the increase in velocity experimentally found by Cheng [16] in swirl stabilised flame $\left(u_{b_{+\infty}} / u_{u-\infty}=3\right)$ and assuming $d \bar{c} / d x$ and $d \bar{u} / d x$ to be distributed 


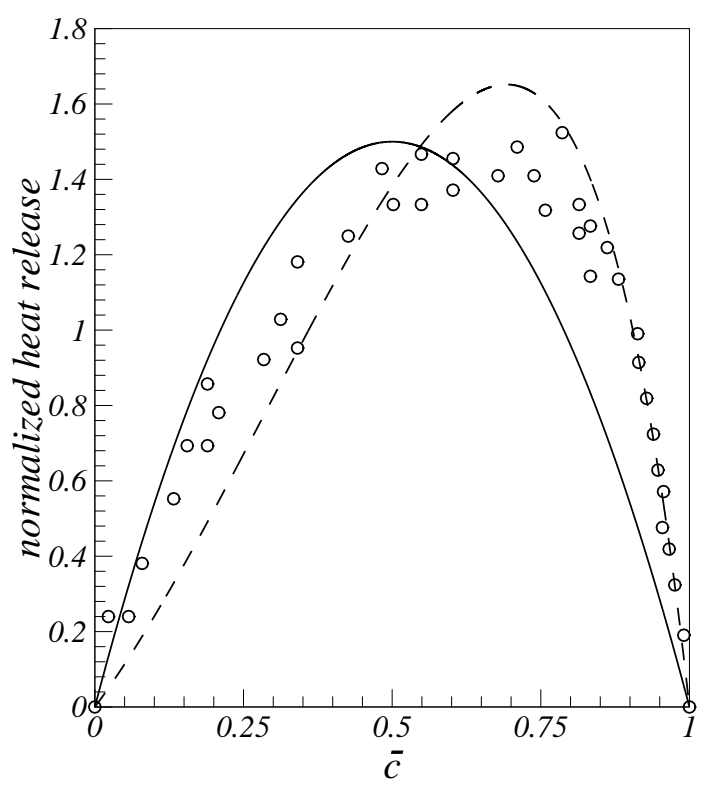

Figure 4. Comparison with distribution of heat release across flame brush from Cheng experiments [11]. _ symmetric BML distribution, $O$ from experimentally measured crossing frequency, - - - present work based on the accurate (gasdynamics) model.

according to the symmetrical distribution $\bar{c}(1-\tilde{c})$, consistently with the assumption in [16]. This distribution, in fact, doesn't differs substantially from the Gaussian one previously assumed here. Figure 4 shows the heat release distributions versus $\bar{c}$. The symmetric distribution from the BML theory is also shown. The figure clearly shows the skewness of the experimental heat release with respect to the theoretical symmetric distribution. As shown in figure 4, the model gives a heat release distribution which is skewed with respect to the symmetric one in a fashion similar to the experimental data. More detailed and quantitatively accurate analysis of these interesting experimental data will be subject of future work.

We believe anyway that this is a remarkable result which, even if on a qualitative basis, shows that that the capability to correctly account for counter-gradient transport directly affect the accuracy we can estimate heat release. In some sense this experimental data and the successfull agreement predicted by our model strongly support our idea that heat release and counter-gradient transport are intimately connected.

\subsection{Validation with experiments for a confined turbulent premixed flame by Frank et al}

The schematics of these experiments is shown in figure $2 \mathrm{~b}$ ). The fuel used is natural gas and the fuel/air mixture flows out fully premixed from a $36 \mathrm{~mm}$ diameter piloted axisymmetric burner. The pilot gases have a very small flow rate (approx. $1.5 \%$ by volume of the main flow rate) and the co-flowing air has a velocity of $2.4 \mathrm{~m} / \mathrm{s}$. A perforated plate with $4-\mathrm{mm}$ diameter holes spaced $7 \mathrm{~mm}$ apart is placed inside the 
burner to generate turbulence. Experimental measurements have been taken for five different flames corresponding to five different values of the ratio $u^{\prime} / s_{L}$. This ratio has been adjusted generally changing the equivalence ratio of the fuel/air mixture (changing the laminar flame speed $s_{L}$ ). The characteristic data of these five flames are reported in Table 1.

In these experiments digital Particle Image Velocimetry (PIV) and Planar LaserInduced Fluorescence (PLIF) are used to provide simultaneous two-dimensional measurements of the velocity and the $O H$ radicals concentration. Sharp gradients in the $O H$ concentration are used to determine the instantaneous location of the flame front.

The radial and axial components of the conditionally averaged velocities and the Reynolds average progress variable $\bar{c}$ have been measured at 40 locations $(5$ axial $\times$ 8 radial) within a $2.8 \times 4.2 \mathrm{~mm}^{2}$ region of the flame located approximately $2.7 \mathrm{~cm}$ downstream of the burner mouth (see figure $2 \mathrm{~b}$ ).

The flames are generally parallel to the burner axis (exception applies mainly to flame $A$ which is characterised by the largest $U_{t}$ ). This is confirmed by the grouping of experimental data points at eight different values of $\bar{c}$ corresponding to eight radial locations within the imaged area. The 1-D theory developed in the present work has been therefore applied here in the direction normal to the flame which in first approximation corresponds to the radial direction.

Flames $B, C, D$ and $E$ have been simulated using a commercial finite volume code where the TFC combustion model and the postprocessing for extracting the total progress variable flux have been implemented. Modelling of turbulent transport is based on a standard $k-\epsilon$ model. The computational domain has been discretised with approx. 15,000 computational cells. Model (31) has been used for calculating the flame stretch factor $G$ in the turbulent flame speed. The critical strain rate has been assumed equal to the inverse of the chemical time scale, i.e. $g_{c r}=s_{L}^{2} / \chi$.

Turbulent scalar transport both of gradient and counter-gradient type was previously observed using DNS. Counter-gradient transport was observed for example in the simulations by [29] where $u^{\prime} / s_{L}=1$ while gradient transport was obtained by Trouvé and Poinsot [30] with $u^{\prime} / s_{L}=10$. Frank et al work is the first experimental evidence of the possibility to have a transition from counter-gradient to gradient scalar transport in turbulent premixed flames. In general the experiments show that this transition occurs when the ratio $u^{\prime} / s_{L}$ decreases. This is valid for example in the case of flames $B, C, D$ as can be seen from figure 5 and Table 1 . According to the modelling ideas proposed here, it should anyway be observed that the $u^{\prime} / s_{L}$ ratio is not sufficient to determine the type of transport and in what extent one component of the total turbulent scalar flux dominates over the other. This is clear from (12) which shows that the gradient part of transport depends also from the actual thickness of the turbulent flame brush (i.e. from $d \tilde{c} / d n$ ). This is also confirmed by the experiments: flame $E$ in fact is characterised by a ratio $u^{\prime} / s_{L}$ which is (slightly) larger than in the case of flame $B$ but with a total turbulent scalar flux which is more biased toward the counter-gradient 

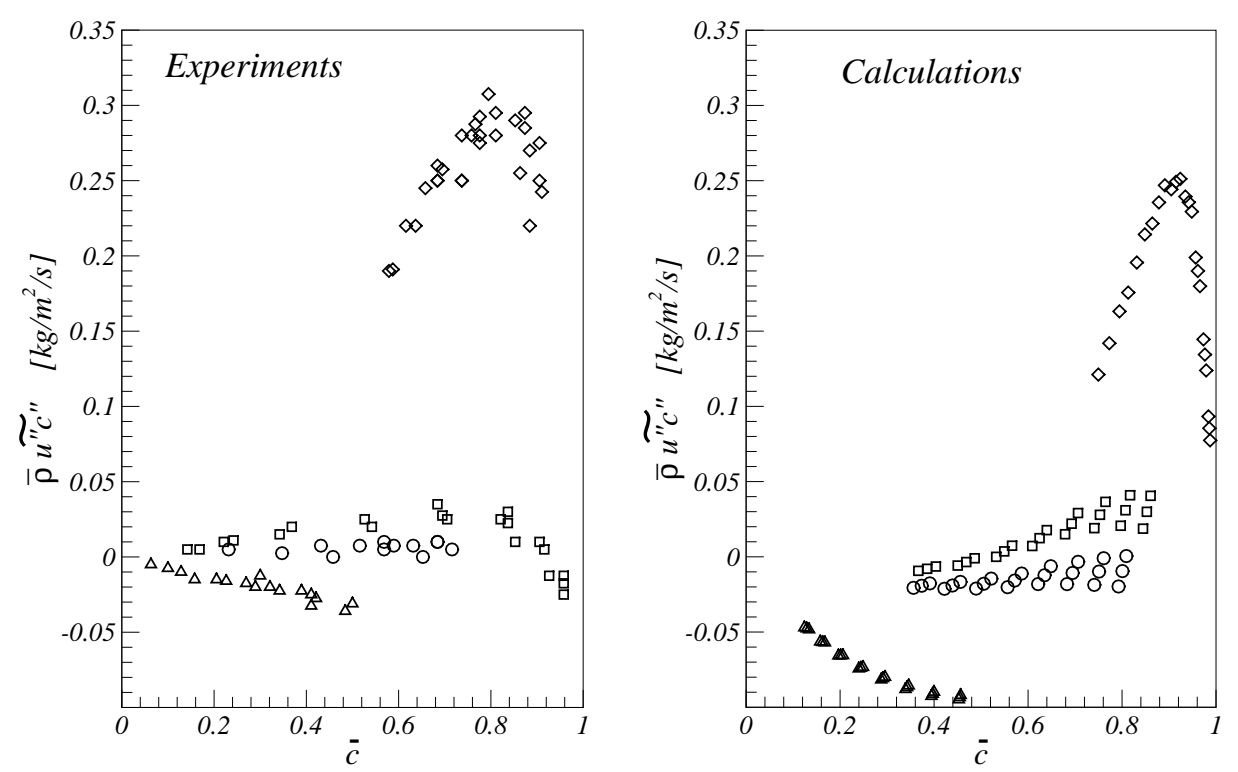

Figure 5. Comparison with experiments from Frank et al [3]. Progress variable flux. $\triangle$ flame $\mathrm{D}, \mathrm{O}$ flame $\mathrm{C}, \square$ flame B, $\diamond$ flame E. Left: experiments. Right: present calculations based on the accurate (gasdynamics) model.

type. An observation of the experimental data shows that flame $E$ is characterised by a substantially larger flame thickness and therefore smaller $d \tilde{c} / d n$ than flame $B$. This explain why, despite the slightly larger value of $u^{\prime} / s_{L}$, flame $E$ has an overall transport more biased toward counter-gradient than flame $B$.

The transition from counter-gradient to gradient type of transport occurs in the experiments in correspondence of Flame $C$, between flame $B$ (counter-gradient) and Flame $D$ (gradient). This transition occurs instead in the numerical simulations between Flame $B$ (mainly counter-gradient) and Flame $C$ (mainly gradient). The predicted flux in Flame $D$ is totally of gradient type and it overpredicts the gradient flux shown by the experiments.

Figures 6 and 7 shows the comparison of conditional velocities. These data give the same information provided by the scalar flux under a different point of view, $\bar{u}_{b}>\bar{u}_{u}$ meaning counter-gradient type of transport and $\bar{u}_{b}<\bar{u}_{u}$ meaing gradient type.

The transition from counter-gradient to gradient type of scalar transport is finally enphasised in figure 8. This figure shows progress variable contours and region where the scalar flux is of gradient type for flames $E, B, C, D$. The figure shows that transport in Flame $\mathrm{E}$ is nearly everywhere of the counter-gradient type with only a small gradient type region existing near the splitter plate where the progress variable attains its maximum gradient. The region of gradient transport increases in size for flame $B$ and $C$ and becomes very broad in the case of flame $D$ because of the very lean conditions of this flame. 

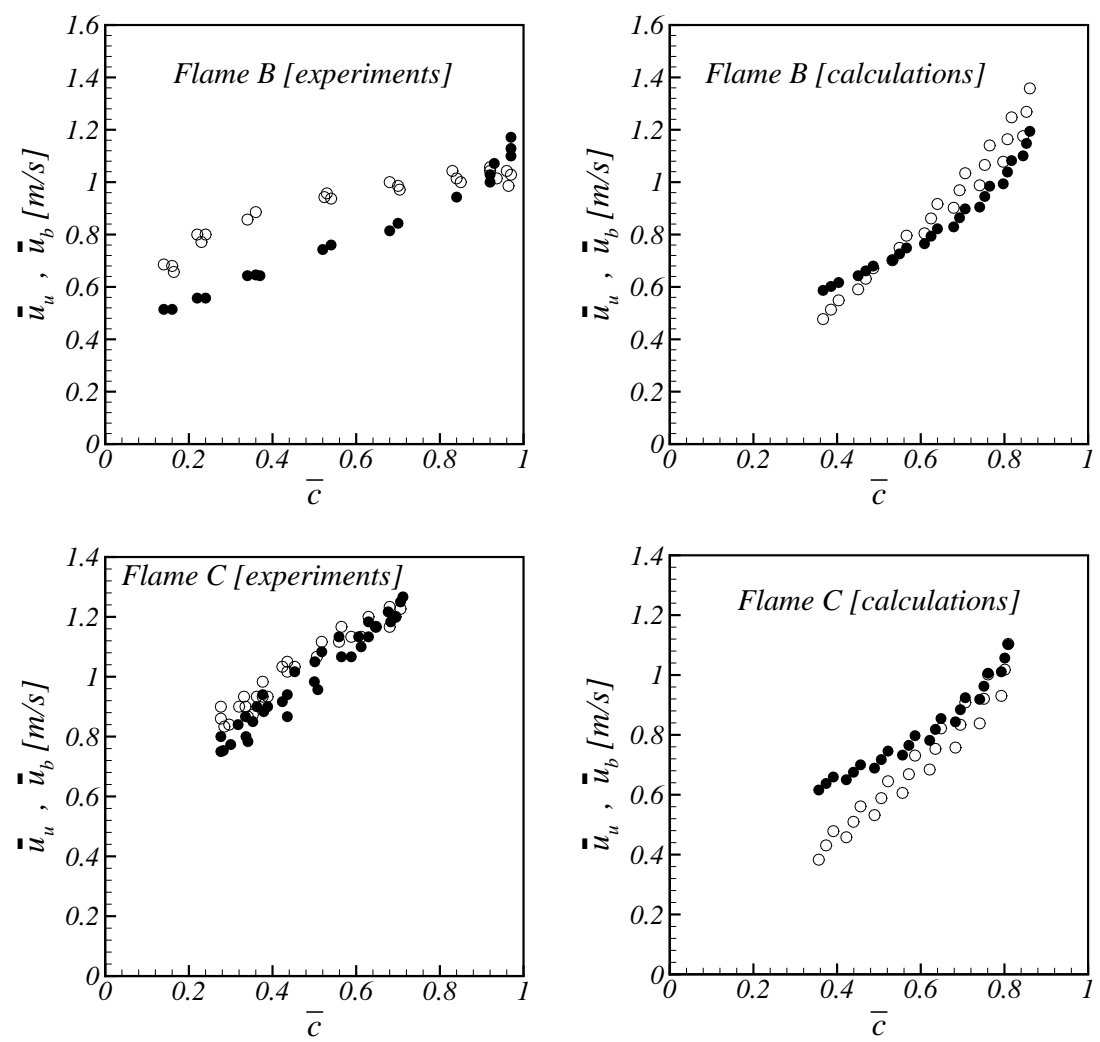

Figure 6. Comparison with experiments from Frank et al [3]. Conditional velocities in flames $B$ and $C$. $\bullet \bar{u}_{u}, \bigcirc \bar{u}_{b}$ Left: experiments; right: present calculations based on the accurate gas dynamics model.

Table 1. Flame parameters in experiments by Frank et al [3].

\begin{tabular}{cccccc}
\hline Flame & $\phi$ & $\Theta$ & $u^{\prime}(\mathrm{m} / \mathrm{s})$ & $u^{\prime} / \bar{u}$ & $u^{\prime} / s_{L}$ \\
\hline $\mathrm{A}$ & 1.00 & 6.5 & 0.86 & 0.16 & 2.3 \\
$\mathrm{~B}$ & 0.70 & 5.2 & 0.53 & 0.10 & 3.1 \\
$\mathrm{C}$ & 0.70 & 5.2 & 0.83 & 0.17 & 4.9 \\
$\mathrm{D}$ & 0.61 & 4.6 & 0.79 & 0.17 & 8.8 \\
$\mathrm{E}$ & 1.30 & 5.9 & 0.85 & 0.17 & 3.6 \\
\hline
\end{tabular}

To close this section we want to enphasise that the theory of the TFC combustion models predicts the existence of thin flamelets (not necessarily laminar) also at much higher $R_{t}$ numbers typical in large-scale and high velocities industrial burner. We expect therefore counter-gradient transport and the transition to gradient also in this case. Regarding this, numerical simulation of Moreau experiments [23] for high velocity premixed combustion performed in [8] using the model based on $\bar{u}_{u}, \bar{u}_{b}=$ const which gives the upper estimation of the counter-gradient transport component, have shown the presence of counter-gradient transport practically along the whole length of the flame. We did not focus in that paper on the transition effect but it is pertinent to note here 

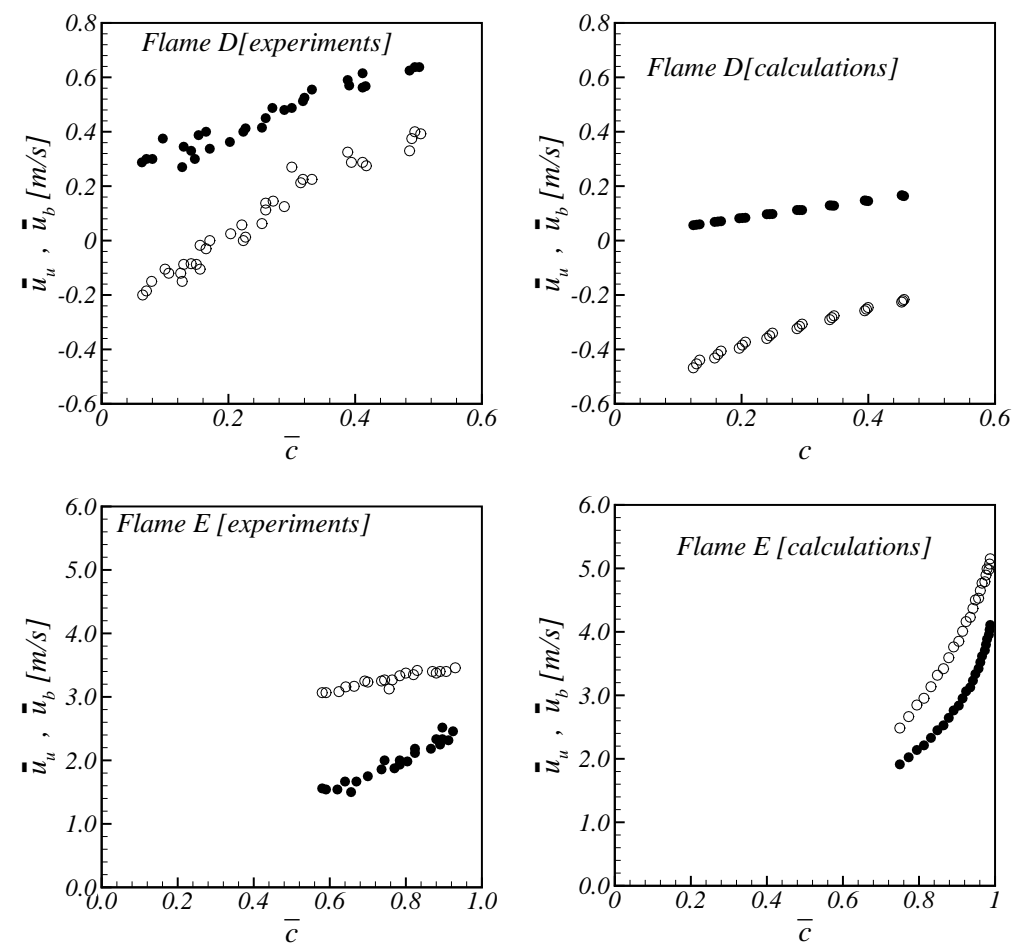

Figure 7. Comparison with experiments from Frank et al [3]. Conditional velocities in flames $D$ and $E$. $-\bar{u}_{u}, \bigcirc \bar{u}_{b}$ Left: experiments; right: present calculations based on the accurate (gasdynamics) model.
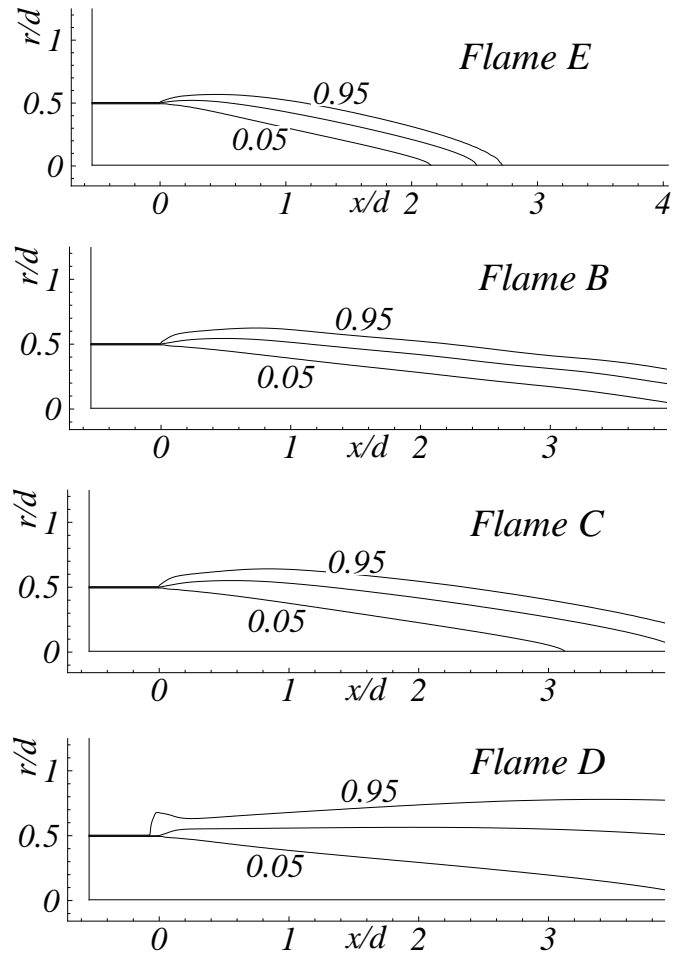
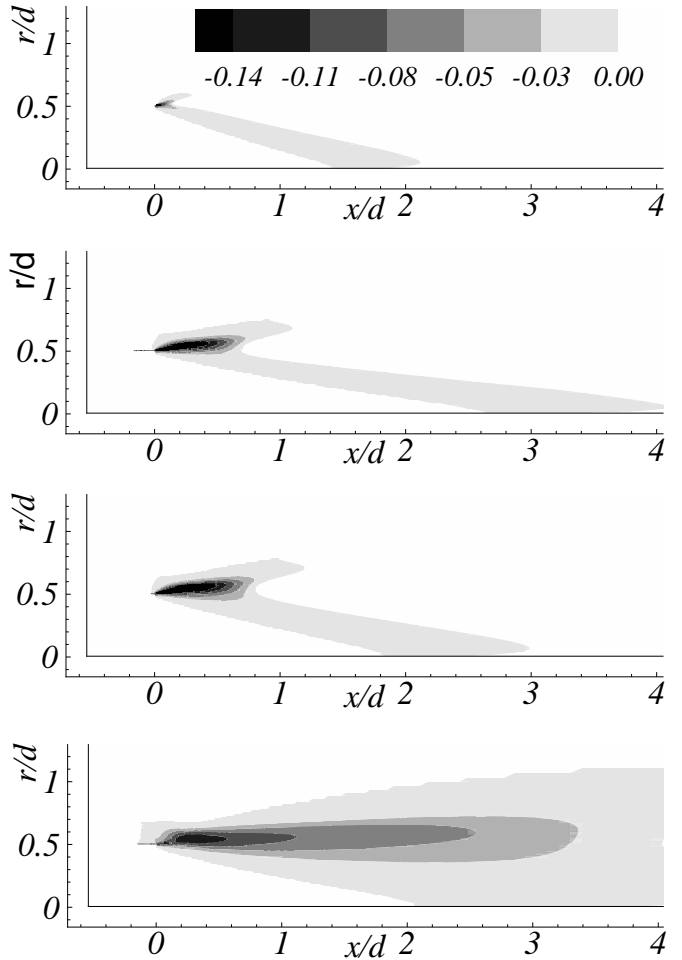

Figure 8. Left: calculated contours of progress variable in flames $B, C, D$ and $E$ in [3] experiments. Right: dark area indicates region of gradient diffusion. 
that in those simulations gradient transport took place only in a small region at the beginning of the flame and transition to counter-gradient transport occured at distance $\sim 1 \mathrm{~cm}$ from the splitter plate anchoring the flame.

\section{Conclusions}

The phenomenon of scalar transport in turbulent premixed flames has been analyzed in the present paper. The analysis starts from the idea (which is consistent with experimental observations) that turbulence has the effect to increase the thickness of the turbulent flame brush in the same fashion it can increase the thickness of a non reactive mixing layer. This happens independently of the nature of the overall progress variable flux (gradient or counter-gradient). Starting from this assumption, a theory on turbulent premixed flames has been developed in a very natural way in the framework of the Turbulent Flame Closure (TFC) model. This theory shows that, despite what commonly believed, the use of gradient transport (based on $k-\epsilon$ modelling for example) to model the turbulent diffusion of the progress variable flux in premixed flames is not in contradiction with the phenomenon of counter-gradient transport. It has been demonstrated in fact that transport in turbulent premixed flames is characterised by two components: real turbulent diffusion which is responsible for thickening the flame brush and gasdynamics transport which is connected with the pressure variation across the turbulent flame brush (counter-gradient in the case of opne flames analysed here).

In particular, the analysis shows that the common terminology "counter-gradient turbulent diffusion in premixed flames" and consequently the possibility to have flames with decreasing thickness are improper and mainly related to a (we believe non correct) interpretation of this transport as pure turbulent diffusion flux.

Two models have been developed for the counter-gradient component using assumptions on the distribution of conditional pressures within the unburnt and burnt mixtures. The first (quite unrealistic) is based on assuming the conditional velocities to be uniform within the turbulent flame brush, i. e. this model doesn't take into account the mutual interaction of cold and hot volums. This model gives principally the possible upper estimation of counter-gradient transport that in typical situations is approximately three times larger than experimentally determined counter-gradient fluxes [1]. The second much more realistic model takes into account the actual interaction (acceleration of slower cold volumes by faster hot volumes and viceversa) via the assumption that conditional pressures are equal $\overline{p_{u}}(x)=\overline{p_{b}}(x)=\bar{p}$. This model gives counter-gradient transport which is in good agreement with Moss experimental data [1].

An important result of the present analysis is that the distribution across the turbulent flame brush of the average heat release is strongly linked with the countergradient transport component. The first model proposed for counter-gradient transport, for example, gives a heat release distribution versus $\bar{c}$ which is symmetrical with respect $\bar{c}=0.5$ for a symmetrical distribution of $d \bar{c} / d x$. The second model gives instead an 
averaged heat release distribution that is skewed toward $\bar{c}>0.5$. Such skewed heat release distribution is confirmed by the experimental data by Cheng et al [11,16] where the flamelet crossing frequency has been analysed.

Finally, from the practical point of view, the present analysis shows that quantitatively correct modeling of counter-gradient transport is possible without the use of the second order moment closures transport models. The combination of the $k-\epsilon$ model for the gradient turbulent transport component and a gasdynamics model for counter-gradient component results in fact in the satisfactory prediction of the countergradient transport phenomenon and of the transition into gradient transport observed experimentally by Frank et al when changing the $u^{\prime} / s_{L}$ ratio.

\section{References}

[1] Moss J B 1980 Simultaneous measurements of concentration and velocity in a open premixed turbulent flame Combust. Sci. Technol. 99115

[2] Li S C, Libby P A and Williams F A 1994 Experimental investigation of a premixed flame in an impinging turbulent stream, 26th Int. Symp. on Combustion (The Combustion Institute) p 1207

[3] Frank J H, Kalt P A M and Bilger R W 1999 Measurement of conditional velocities in turbulent premixed flames by simultaneous OH PLIF and PIV Combust. Flame 116220

[4] Zimont V L 1979 The theory of turbulent combustion at high Reynolds numbers Combust. Expl. and Shock Waves 15305

[5] Zimont V L 1999 Gas premixed combustion at high turbulence. Turbulent Flame Closure combustion model. Proc. of the Mediterranean Combustion Symposium Antalya Turkey p 1155

[6] Dinkelacker F, Soika A, Most A and H" oller A 1998 Measurement of local temperature gradients in turbulent combustion systems using two-dimensional laser diagnostics Proc. 11th International Heat Transfer Conference

[7] Chen Y and Mansour M S 1998 27th Int. Symp. on Combustion (The Combustion Institute)

[8] Zimont V L, Biagioli F and Syed K J 2000 Modelling turbulent premixed combustion in the intermediate steady propagation regime Int. J. of Progress in Computational Fluid Dynamics 1 No. $1 / 2 / 3,14$

[9] Prudnikov A G, Volinsky M S, Sagalovich V N 1971 Processi smeseobrazovaniya i goreniya v vozdushno-reaktivnih dvigatelyah Moscow (in Russian)

[10] Zimont V L, Polifke W, Bettelini M and Weisenstein W 1998 J. of Engineering for Gas Turbines (Transaction of the ASME) $\mathbf{1 2 0}$ p 526

[11] Cheng R K and Sheperd I G 1991 The influence of burner geometry on premixed turbulent flame propagation Combust. Flame, 85, 7

[12] Veynante D, Trouvé A, Bray K N C and Mantel T 1997 Gradient and counter-gradient scalar transport in turbulent premixed flames J. Fluid Mech., 332263

[13] Bray K N C, Libby P A, Masuya G and Moss J B 1981 Turbulence production in premixed turbulent flames Combust. Sci. and Technol. 25127

[14] Libby P A and Bray K N C 1981 Countergradient diffusion in premixed turbulent flames, AIAA $J ., 19(2)$ p 205

[15] Prudnikov A G 1958 Flame Turbulence 7th Int. Symp. on Combustion (The Combustion Institute) p 575

[16] Cheng R K 1995 Velocity and scalar characteristics of premixed turbulent flames stabilised by weak swirl Combust. Flame, 101, 1

[17] Zimont V L 1996 2nd answer to F. A. Williams comment to paper [27] 26th Int. Symp. On Combustion (The Combustion Institute) p 256 
[18] Karpov V P, Lipatnikov A N and Zimont V L 1994 A model of premixed turbulent combustion and its validation Archivium Combustionis, 4, 125

[19] Lipatnikov A 1996 1st answer to F. A. Williams comment to paper [27] 26th Int. Symp. On Combustion (The Combustion Institute) p 256

[20] Damkohler G 1947 NACA Tech. Memo. 1112

[21] Shchelkin K I 1947 NACA Tech. Memo. 1110

[22] Bray K N C and S Cant 2000 personal communication (paper title will be provided later) Combustion and Flame 120(1-2) 1-18

[23] Moreau P 1977 Turbulent flame development in a high velocity premixed flow AIAA paper N. 7749

[24] Bradley D, Lau A K C and Lawes M 1992 Phil. Trans. Royal Soc. 333(A), 359

[25] Zimont V L and Lipatnikov A N 1995 A numerical model of premixed turbulent combustion of gases Chem. Phys. Reports, 14(7) 993

[26] Bray K N C 1987 Methods of including realistic chemical reaction mechanisms in turbulent combustion models Complex chemical reactions systems. Mathematical modelling and simulation, (Heidelberg:Springer) p 356

[27] Karpov V P, Lipatnikov A N and Zimont V L 1996 A test of an engineering model of premixed turbulent combustion 26th Int. Symp. on Combustion (The Combustion Institute) p 249

[28] Bray K N C, Champion M and Libby P A 1988 Mean reaction rates in premixed turbulent flames 20nd Int. Symp. on Combustion (The Combustion Institute) p 763

[29] Zhang S and Rutland C J 1995 Premixed flame effects on turbulence and pressure-related terms Combust. Flame 102447

[30] Trouvé A and Poinsot T 1994 The evolution equation for the flame surface density in turbulent premixed combustion J. Fluid Mech. 2781 